\title{
Geometric Realization of Simplicial Complexes
}

\author{
Patrice Ossona de Mendez ${ }^{1}$
}

CNRS UMR 8557 - E.H.E.S.S., 54 Bd Raspail, 75006 Paris, France

pom@ehess.fr

\begin{abstract}
We show that an abstract simplicial complex $\Delta$ may be realized on a grid of $\mathbb{R}^{d-1}$, where $d=\operatorname{dim} P(\Delta)$ is the order dimension (Dushnik-Miller dimension) of the face poset of $\Delta$.
\end{abstract}

\section{Introduction}

Abstract simplicial complexes are related to order dimension in Section 2 through the complex of a $d$-representation. This construction is analogous to the one introduced by Dushnik in [10] and similar to the one used by Scarf in mathematical economy [14] under the name of primitive sets and which has been applied to integer programming by Barany, Howe and Scarf [1] and to commutative algebra by Bayer, Peeva and Sturmfels [2]. In Section 3, we give a simple necessary and sufficient condition for a mapping to be a geometric realization of an abstract simplicial complex. This characterization leads in Section 4 to the geometric realization of the abstract simplicial complex defined by a $d$-representation on a grid in $\mathbb{R}^{d-1}$. This generalizes the result of Schnyder on planar graphs [15] (see also [3][4]). In Section 5, we prove that any abstract simplicial complex may be triangulated into a "standard" $d$-representation having the same face poset dimension. In Section 6, we extend the vertex shelling order introduced earlier by Fraysseix, Pach and Pollack for planar triangulations [8] and show that a complex is vertex-shellable if and only if it is shellable (in the usual sense). We also prove that a vertex shelling order of the triangulation $\Delta^{+}$mentioned above may be easily derived from the "generating" total orders, generalizing a result proved in [5]. Eventually, Theorem 7 gathers most of the main results of the paper in a single statement.

We recall some basic definitions on simplicial complexes. For further information, see [12]. In the following, we will consider only finite simplicial complexes, what will justify the following definition. An abstract simplicial complex $\Delta$ is a non-empty finite collection of finite sets such that $X \in \Delta, Y \subseteq X$ implies $Y \in \Delta$. The union $V(\Delta)$ of the members of $\Delta$ is the vertex set of $\Delta$. The members of $\Delta$ are the faces of $\Delta$. The dimension of a face $X$ of $\Delta$ is $\operatorname{dim} X=|X|-1$. The dimension $\operatorname{dim} \Delta$ is the maximum dimension of any face of $\Delta$. If $\Delta$ and $\Delta^{\prime}$ are abstract simplicial complexes with disjoint ground sets, we recall that their combinatorial join is the abstract simplicial complex $\Delta * \Delta^{\prime}$ defined by $\Delta * \Delta^{\prime}=\left\{X \cup X^{\prime}, \quad X \in \Delta, X^{\prime} \in \Delta^{\prime}\right\}$. By extension, $\Delta * p$ will denote $\Delta *\{\emptyset,\{p\}\}$. An abstract simplicial complex $\Delta$ is pure if all the maximal faces of $\Delta$ (with respect to inclusion) have the same dimension, that is if any face of $\Delta$ is included in 
a face of $\Delta$ with dimension $\operatorname{dim} \Delta$. The face poset $P(\Delta)$ of $\Delta$ is the poset which consists of all the faces of $\Delta$ ordered by inclusion. For a poset $P$, a realizer of $P$ is a set of total orders whose intersection is $P$. The minimum cardinality of a realizer of poset $P$ is its Dushnik-Miller dimension (or simply the dimension) $\operatorname{dim} P[11]$.

\section{The Complex of a $d$-Representation}

Let $d>0$ be an integer and let $V$ be a finite set. A $d$-representation $R=\left(<_{1}\right.$ $\left., \ldots,<_{d}\right)$ of $V$ is a set of $d$ total orders on $V$ whose intersection is an antichain. With respect to $R$, the supremum section $S(X)$ of a subset $X \subseteq V$ is the subset of $X$ whose elements are the maxima of $X$ for some linear order in $R$ :

$$
S(X)=\left\{x \in X, \quad \exists 1 \leq i \leq d, \forall y \in X-\{x\}, x>_{i} y\right\}
$$

Given a $d$-representation $R$ of $V$, the complex of $R$ is the set $\Sigma(R)$ of all the subsets $X$ of $V$ such that $\forall v \in V, v \in S(X \cup\{v\})$

Lemma 1. The complex $\Sigma(R)$ of a d-representation $R$ of $V$ is an abstract simplicial complex.

Proof. It is straightforward that $S(A) \supseteq S(B) \cap A$ whenever $A \subseteq B$. Thus, if $X^{\prime}$ is a subset of a set $X \in \Sigma(R)$, we get $v \in S\left(X^{\prime} \cup\{v\}\right)$ for any element $v \in V$ as $v \in S(X \cup\{v\})$.

Theorem 1. Let $(V, \Delta)$ be an abstract simplicial complex with vertex set $V$.

Then, $\operatorname{dim} P(\Delta)$ is the smallest integer $d$, such that $\Delta$ is a subcomplex of some d-representation of $V$.

Proof. $\quad-\Delta$ is a subcomplex of a $\operatorname{dim} P(\Delta)$-representation of $V$ :

Consider a realizer $\prec_{1}, \ldots, \prec_{d}$ of cardinality $d=\operatorname{dim} P(\Delta)$ of $P(\Delta)$ and let $R=\left(<_{1}, \ldots,<_{d}\right)$ be the $d$-representation of $V$ induced by the restrictions on $V$ of the $d$ total orders $\prec_{1}, \ldots, \prec_{d}$.

Let $X$ be an element of $\Delta$. Then, for all $1 \leq i \leq d$ and all $x \in X$ we have $x \prec_{i} X$, as $X$ is, by definition, greater than its elements in the face poset. Moreover, $X$ is not comparable to any element which does not belong to $X$. Hence, for any $y \notin X, \exists 1 \leq i \leq d, \forall x \in X, \quad x \prec_{i} y$. Hence, $\forall y \notin X, y \in$ $S(X+y)$. Similarly, if $x$ belongs to $X$, either $X=\{x\}$ and $x \in S(X)$ or $X-x$ is a simplex which belongs to $\Delta$ and hence $x \in S(X-x+x)$ and thus $x \in S(X)$. Altogether, $X$ belongs to $\Sigma(R)$.

- If $\Delta$ is a subcomplex of a $d$-representation of $V$, then $d \geq \operatorname{dim} P(\Delta)$ :

Insert in the $d$ total orders of the representation the faces of $\Delta$ (different from vertices) the following way: In the linear order $<_{i}$, insert just after the vertex $x$ all the faces including $x$ and vertices smaller than $x$ (with respect to linear order $<_{i}$ ), sorted by increasing size and, for a same size, in lexicographic order (with respect to $<_{i}$ ). Then, the face-inclusion of $X$ in $Y$ 
in $\Delta$ obviously correspond to $X<_{i} Y$ (for all $1 \leq i \leq d$ ). Otherwise, if $X$ and $Y$ are not compared in $P(\Delta)$, there exists $a \in X \backslash Y$ and $b \in Y \backslash X$. As $\Delta$ is a subcomplex of the $d$-representation, there exists a linear order $i$ such that $a$ is greater than all the vertices of $Y$ with respect to $<_{i}$. Hence, $X>_{i} Y$. Similarly, there exists $j$ such that $Y>_{j} X$. Thus $X$ and $Y$ are not comparable in the intersection of the $d$ total orders. Altogether, $P(\Delta)$ is equal to the intersection of the $d$ total orders and hence $\operatorname{dim} P(\Delta) \leq d$.

\section{Geometric Realizations of Simplicial Complexes}

Consider an injective mapping $f: V(\Delta) \rightarrow \mathbb{R}^{n}$ (this mapping is naturally extended to map the subsets of $V(\Delta)$ to the corresponding subsets of $\mathbb{R}^{n}$ ). We shall say that $f$ is a geometric realization of $\Delta$ in $\mathbb{R}^{n}$ if $f(\Delta)$ is a geometric simplicial complex, i.e. if

- for any face $X$ of $\Delta, f(X)$ is a set of affinely independent points (i.e. defines a simplex) of $\mathbb{R}^{n}$,

- the intersection of two faces of $f(\Delta)$ is a face of $f(\Delta)$, that is, for any faces $X$ and $Y$ of $\Delta$ :

$$
\operatorname{Conv}(f(X)) \cap \operatorname{Conv}(f(Y))=\operatorname{Conv}(f(X) \cap f(Y))
$$

where $\operatorname{Conv}(P)$ denotes the convex hull of the point set $P$.

If $V(\Delta)$ is a set of points in $\mathbb{R}^{n}, \Delta$ is thus a geometric simplicial complex if and only if the identity is a geometric realization of $\Delta$. We shall say that an abstract simplicial complex $\Delta$ is realizable in $\mathbb{R}^{n}$ if there exists a geometric realization of $\Delta$ in $\mathbb{R}^{n}$. We remark that the first of the two conditions we gave for $f$ to be a geometric realization implies $n \geq \operatorname{dim} \Delta$.

Lemma 2. If $\operatorname{Conv}(f(X)) \cap \operatorname{Conv}(f(Y))=\operatorname{Conv}(f(X) \cap f(Y))$ holds for any two faces $X$ and $Y$ of an abstract simplicial complex $\Delta$, then $f$ is a geometric realization of $\Delta$.

Proof. The injectivity of $f$ is straightforward as $\operatorname{Conv}(f(\{x\})) \cap \operatorname{Conv}(f(\{y\}))=$ $\emptyset$ whenever $x \neq y$. Thus, we only have to prove that the image of a set $X \in \Delta$ is a set of affinely independent points. Let us prove it ad absurdum. Assume $x_{1}, \ldots, x_{k}$ are elements of $X$ having linearly dependent images by $f$. Up to a relabeling of the $x_{i}$, we may assume that there exists an integer $1 \leq a<k$, and real numbers $\lambda_{1}, \ldots, \lambda_{k-1}$, such that $f\left(x_{k}\right)=\sum_{i=1}^{k} \lambda_{i} f\left(x_{i}\right), \sum_{i=1}^{k} \lambda_{i}=1$, $\lambda_{1}, \ldots, \lambda_{a}$ are negative and $\lambda_{a+1}, \ldots \lambda_{k-1}$ are positive.

Then, define $\alpha=\sum_{i=a+1}^{k} \lambda_{i}$. As the $\lambda_{i}$ sum up to 1 , all the $\lambda_{i}$ are not negative and $\alpha>0$. Hence, we have:

$$
\frac{1}{\alpha} f\left(x_{k}\right)+\sum_{i=1}^{a}\left(-\frac{\lambda_{i}}{\alpha}\right) f\left(x_{i}\right)=\sum_{i=a+1}^{k-1} \frac{\lambda_{i}}{\alpha} f\left(x_{i}\right)
$$


Thus, $\operatorname{Conv}\left(f\left(\left\{x_{1}, \ldots, x_{a}, x_{k}\right\}\right)\right) \cap \operatorname{Conv}\left(f\left(\left\{x_{a+1}, \ldots, x_{k-1}\right\}\right)\right)$ is not empty although the faces $\left\{x_{1}, \ldots, x_{a}, x_{k}\right\}$ and $\left\{x_{a+1}, \ldots, x_{k-1}\right\}$ of $\Delta$ (sub-faces of $X$ ) are disjoint; we are led to a contradiction.

Lemma 3. If $\operatorname{Conv}(f(X)) \cap \operatorname{Conv}(f(Y))$ is empty for any two disjoint faces $X$ and $Y$ of an abstract simplicial complex $\Delta$, then $\operatorname{Conv}(f(X)) \cap \operatorname{Conv}(f(Y))=$ $\operatorname{Conv}(f(X) \cap f(Y))$ holds for any two faces $X$ and $Y$ of $\Delta$.

Proof. Let $X$ and $Y$ be any two non-disjoint faces of $\Delta$ and let $\pi$ be a point in $\operatorname{Conv}(f(X)) \cap \operatorname{Conv}(f(Y))$. The point $\pi$ may be expressed as a weighted average of the points in $f(X): \pi=\sum_{x \in X} \alpha(x) f(x)$, where $\alpha$ is a mapping from $V(\Delta)$ to $\mathbb{R}^{+}$with sum 1 ans support $\operatorname{Supp}(\alpha) \subseteq X$. Similarly, $\pi=\sum_{y \in Y} \beta(y) f(y)$, where $\beta$ is a function from $V(\Delta)$ to $\mathbb{R}^{+}$with sum 1 and $\operatorname{support} \operatorname{Supp}(\beta) \subseteq Y$. Let $\lambda(z)=\min (\alpha(z), \beta(z))$. This function is positive and has support $\operatorname{Supp}(\lambda) \subseteq$ $X \cap Y$. Let $s$ be its sum: $s=\sum_{z \in X \cap Y} \lambda(z)$. Let us show that $s$ cannot be different from 1: otherwise,

$$
\sum_{x \in X}\left(\frac{\alpha(x)-\lambda(x)}{1-s}\right) f(x)=\sum_{y \in Y}\left(\frac{\beta(y)-\lambda(y)}{1-s}\right) f(y)
$$

and, if $X^{\prime}$ denotes the support of $\alpha-\lambda$ and $Y^{\prime}$ denotes the support of $\beta-\lambda$, $\operatorname{Conv}\left(f\left(X^{\prime}\right)\right) \cap \operatorname{Conv}\left(f\left(Y^{\prime}\right)\right)$ is not empty although the faces $X^{\prime} \subseteq X$ and $Y^{\prime} \subseteq Y$ are disjoint. Hence, $s=1$, what may only be achieved by $\alpha=\beta$. Thus, $\pi$ belongs to $\operatorname{Conv}(f(X) \cap f(Y))$.

Theorem 2 (Folklore). Let $\Delta$ be an abstract simplicial complex and let $f$ : $V(\Delta) \rightarrow \mathbb{R}^{n}$ be a mapping. Then, $f$ is a geometric realization of $\Delta$ in $\mathbb{R}^{n}$ if and only if $\operatorname{Conv}(f(X))$ and $\operatorname{Conv}(f(Y))$ are disjoint, for any two disjoint faces $X, Y$ of $\Delta$.

Proof. If $f$ is a geometric realization then disjoint sets in $\Delta$ are maped into disjoint simplices. Conversely, if disjoint faces are maped into set having disjoint convex hulls, then the intersection of the convex hulls of the images of any two faces $X$ and $Y$ of $\Delta$ is the convex hull of the image of their intersection, according to Lemma 3. Then, according to Lemma $2, f$ is a geometric realization of $\Delta$ in $\mathbb{R}^{n}$.

\section{Geometric Realization of a $d$-Representation}

In the following, we consider a $d$-representation $R=\left(<_{1}, \ldots,<_{d}\right)$ of a set $V$ and a mapping $f$ from $V$ to $\mathbb{R}^{d}$, such that $f_{i}$ is strictly positive and strictly increasing with respect to $<_{i}$ (for $1 \leq i \leq d$ ). We denote by $P$ the image of $V$ and $\Delta(P)$ the image of $\Sigma(R)$. As $f$ is clearly injective, $\Delta(P)$ and $\Sigma(R)$ are isomorphic. 
Let $X \subseteq P$ be a subset of points, $\sigma(X)$ denotes the point with coordinates $\sigma_{i}(X)=\max _{\pi \in X} \pi_{i}$ and $\Theta(X)$ denotes the closed set $\Theta(X)=\{\pi, \quad \forall 1 \leq i \leq$ $\left.n, \pi_{i} \leq \sigma_{i}(X)\right\}$. According to these definitions and according to the definition of $\Sigma(R)$, a point set $X \subseteq P$ is a face of $\Delta(P)$ if and only if

- any point in $X$ belongs to the frontier of $\Theta(X)$,

- no point of $P$ belongs to the interior of $\Theta(X)$.

Theorem 3. Let $R=\left(<_{1}, \ldots,<_{d}\right)$ be a d-representation of a set $V$. Let $f$ be any mapping from $V$ to $\mathbb{R}^{d}$, such that $f_{i}$ is strictly positive and increasing with respect to $<_{i}$ sufficiently fast (that is: the ratio of consecutive values of $f_{i}$ is bigger than $A \geq 1+\sqrt{d})$, let $\left(\lambda_{1}, \ldots, \lambda_{d}\right)$ be $d$-uple of positive real numbers different from $(0, \ldots, 0)$ and let $H$ be the hyperplane of $\mathbb{R}^{d}$ defined by $\sum_{i} \lambda_{i} x_{i}=1$.

Then, the mapping $\phi: V \rightarrow H$ defined by $\phi_{i}(x)=\frac{f_{i}(x)}{\sum_{j} \lambda_{j} f_{j}(x)}$ is a geometric realization of $\Sigma(R)$ in $H \approx \mathbb{R}^{d-1}$.

Proof. We shall first prove that $\Delta(P) * O$ is a geometric simplicial complex, that is that $\operatorname{Conv}(X) \cap \operatorname{Conv}(Y)$ is empty for any two disjoint sets of $\Delta(P) * O$ (according to Theorem 2). As, for any face $X \in \Delta(P) * O, X \cup\{O\}$ is also a face of $\Delta(P) * O$, it is necessary and sufficient to prove that $\operatorname{Conv}(X \cup\{O\}) \cap \operatorname{Conv}(Y)$ is empty for any two faces $X, Y$ of $\Delta(P)$ or, equivalently, that there exists an hyperplane $H$ passing through $O$ and which separates $X$ from $Y$. As no point of $X$ belongs to $\Theta(Y)$, each point $x \in X$ has a coordinate bigger than the corresponding one of $\sigma(Y)$. Hence, the set $I=\left\{i, \exists x \in X, x_{i}>\sigma_{i}(Y)\right\}$ is not empty. Similarly, the set $J=\left\{j, \exists y \in Y, y_{i}>\sigma_{i}(X)\right\}$ is also not empty. Let $a(x)=\sum_{i \in I} \frac{x_{i}}{\sigma_{i}(Y)}$ and $b(x)=\sum_{j \in J} \frac{x_{j}}{\sigma_{j}(X)}$. We have: $a(x) \geq A$ for any $x \in X$ as there exists an index $i$, for which $x_{i}>1$ and hence $x_{i} \geq A$; To the opposite, $a(y)<1+\frac{d-1}{A}$ for $y \in Y$, as only one coordinate of $y$ may reach the maximum value. Similarly, $b(x)<1+\frac{d-1}{A}$ for any $x \in X$ and $b(y) \geq A$ for any $y \in Y$. As $A \geq 1+\sqrt{d}$, we have $A>1+\frac{d-1}{A}$. Thus, the hyperplane defined by $a(x)=b(x)$ passes through $O$ and separates $X$ (for which $a(x)>b(x)$ from $Y$ (for which $a(x)<b(x))$ and $\Delta(P) * O$ will be a geometric simplicial complex.

Now, consider the hyperplane $H^{\prime}$ defined by $\sum_{i} \lambda_{i} x_{i}=\epsilon$, where $\epsilon$ is a sufficiently small positive real number, so that $H^{\prime}$ separates $O$ from $P$. Then, the intersection of $\Delta(P) * O$ and $H^{\prime}$ is a geometric realization of $\Delta(P) \approx \Sigma(R)$ and so is its homothetic image defined by the image of $\Sigma(R)$ by the mapping $\phi$.

\section{Triangulation}

Lemma 4. Let $R=\left(<_{1}, \ldots,<_{d}\right)$ be a d-representation of $V$ with complex $\Sigma(R)$ and let $x$ be the maximum of $<_{k}$. For $i \neq k$, let $<_{i}^{\prime}$ be the total order on $V$ where $x$ precedes all the elements of $V \backslash\{x\}$ and the element of $V \backslash\{x\}$ are ordered by $<_{i}$. Then $R^{\prime}=\left(<_{1}^{\prime}, \ldots,<_{k-1}^{\prime},<_{k},<_{k+1}^{\prime}, \ldots,<_{d}\right)$ is a d-representation which complex $\Sigma\left(R^{\prime}\right)$ includes $\Sigma(R)$. 
Proof. Denote by $S^{\prime}$ the supremum section corresponding to $R^{\prime}$. Let $X$ be a subset of $V$, then $S(X) \subseteq S^{\prime}(X)$ :

- If $x \notin X, \quad S^{\prime}(X)=S(X)$,

- If $x \in X, \quad S^{\prime}(X)=\{x\} \cup S(X \backslash\{x\}) \supseteq S(X)$

Thus, any element $X$ of $\Sigma(R)$ belongs to $\Sigma\left(R^{\prime}\right)$ : for all $v \in V, v \in S(X \cup\{v\}) \subseteq$ $S\left(X \cup\{v\}\right.$ and hence $X \in \Sigma\left(R^{\prime}\right)$.

A $d$-representation $R=\left(<_{1}, \ldots,<_{d}\right)$ on a set $V$ is standard if $|V| \geq d$ and, for all $i \neq j$, the maximum element of $<_{i}$ is one of the $d-1$ smallest elements of $<_{j}$. The maxima of the orders of a standard $d$-representation are the exterior elements of this representation. The other elements of $V$ are the interior elements. An abstract simplicial complex is standard if it is the complex of some $\operatorname{dim} P(\Delta)$ representation. Notice that, for a standard abstract simplicial complex $\Delta$, we have: $\operatorname{dim} \Delta=\operatorname{dim} P(\Delta)-1$.

Given any subset $X$ of $V$ and a $d$-representation $R$ of $V$, we define the shade function $I_{X}$ of $X$ on $V \backslash X$ as $I_{X}(u)=\left\{i \in[1, d], \forall x \in X, u>_{i} x\right\}$. Given any subset $X$ of $V$ and a $d$-representation $R$ of $V$, we define the shading order $<_{X}$ of $X$ on $V \backslash X$ as follows: $u<_{X} v \Longleftrightarrow\left(I_{X}(u) \subseteq I_{X}(v)\right)$ and $\left(\forall i \in I_{X}(u), u<_{i} v\right)$

Lemma 5. Any standard representation will be pure. More precisely, let $R=$ $\left(<_{1}, \ldots,<_{d}\right)$ be a standard representation. If $X$ is a face of $\Sigma(R)$ which maximal element $x_{1}$ (with respect to $<_{1}$ ) is not an exterior element of $R$, then, for every $\operatorname{dim} X<k<d$ there exists a $k$-dimensional face which includes $X$ and has $x_{1}$ as a maximum element with respect to $<_{1}$.

Proof. Let $X$ be an element of $\Sigma(R)$ different from the $|X|$ first elements of $<_{1}$ and assume $|X|<d$.

If $X$ is included into the set $\left\{v_{1}, \ldots, v_{k}\right\}$ of the external elements of $R$, the addition of any external element smaller or equal to $x_{1}$ with respect to $<_{1}$ will do.

Otherwise, let $x$ be an element of $x$ which is maximal in $X$ with respect to two different total orders and let $<_{k}$ be one of them for which $k \neq 1$. Obviously, $x$ is not an external element of $R$ as an external element is greater than an internal with respect to exactly one total order. For the same reason, $v_{k}$ does not belong to $X$ and is smaller or equal to $x_{1}$ with respect to $<_{1}$. Let $\alpha$ be a minimal element of the set $\left\{v \notin X, v<_{1} x_{1}\right.$ and $\left.v<_{X} v_{k}\right\} \cup\left\{v_{k}\right\}$. As $I_{X}(\alpha) \neq \emptyset$ (otherwise, $\alpha \notin S(X+\alpha)$ would contradict $X \in \Sigma(R))$ and as $I_{X}(\alpha) \subseteq I_{X}\left(v_{k}\right)$ by construction, we get: $I_{X}(\alpha)=\{k\}$. This ensures that $S(X+\alpha)=X+\alpha$. Assume there exists an element $y \notin X+\alpha$, such that $y \notin S(X+\alpha+y)$. As $X \in \Sigma(R)$, the element $y$ belongs to $S(X+y)$. As $I_{X}(\alpha)=\{k\}$, the only possibility for $y$ not to belong to $S(X+\alpha+y)$ corresponds to the situation where $I_{X}(y)=\{k\}$ and $y<_{k} \alpha$, that is: $y<_{X} \alpha$. Moreover, $y$ is smaller or equal to $x_{1}$ with respect to $<_{1}$ (as $\left.1 \notin I_{X}(y)\right)$ and this contradicts the minimality of $\alpha$.

Theorem 4. Any abstract simplicial $\Delta$ complex may be triangulated into a standard representation having the same face poset dimension. 
Proof. As a direct consequence of Theorem 1, $\Delta$ is the subcomplex of some $d$-representation $R_{0}$. By successive applications of Lemma 4 , there exists a standard $d$-representation $R$, such that $\Sigma(R)$ includes $\Sigma\left(R_{0}\right)$ and hence includes $\Delta$. According to Lemma $5, \Sigma(R)$ is pure of dimension $d-1$ and hence is a triangulation of $\Delta$.

\section{$6 \quad$ Shellability of Standard Complexes}

Shellability of pure simplicial complexes has been extensively studied. We shall introduce here the vertex shellability, which is a generalization of the concept introduced in [8] and which has proved its efficiency through numerous applications in quite different kind of problems related to planarity (see, for instance, $[6][7][9][13])$. We shall prove that the concepts of shellability and vertexshellability are actually equivalent. Thatfor, recall that a pure abstract simplicial complex $\Delta$ of dimension $d-1$ is shellable if all its $(d-1)$-faces (that is: all its elements of cardinality $d$ ) can be listed $F_{1}, \ldots, F_{s}$ in such a way that $\left(\bigcup_{i=1}^{j-1} \overline{F_{i}}\right) \cap \overline{F_{j}}$ is pure of dimension $d-2$ for every $1<j \leq s$ (where $\overline{F_{i}}$ is defined by: $\overline{F_{i}}=\left\{X \in \Delta, X \subseteq F_{i}\right\}$ ) or, equivalently, for $1 \leq i<j \leq s$, any subset $X$ of $F_{i}$ and $F_{j}$, there exists a $(d-2)$-dimensional face $Y \supseteq X$ and a $(d-1)$-dimensional face $F_{h}$ (for some $h<j$ ) such that $Y$ is included in both $F_{h}$ and $F_{j}$.

A pure abstract simplicial complex $\Delta$ of dimension $d-1$ is said to be vertex shellable if all its vertices can be listed $v_{1}, \ldots, v_{n}$ in such a way that:

$-\left\{v_{1}, \ldots, v_{d}\right\} \in \Delta$,

- for all face $\sigma \in \Delta$ with maximum vertex $v_{k}(k>d)$, there exists $j<k$, such that $v_{j} \notin \sigma$ and $\left.\sigma-v_{k}+v_{j} \in \Delta\right)$,

- for all vertex $v_{k}(k \geq d)$, the abstract simplicial complex $\Delta_{k}=\left\{\sigma-v_{k}, \sigma \in\right.$ $\Delta$ and $\left.\sigma \subseteq\left\{v_{1}, \ldots, v_{k}\right\}\right\}$ is a pure $d-2$ dimensional shellable simplicial complex.

Lemma 6. Let $\Delta$ be a pure $d-1$ dimensional shellable simplicial complex and let $F_{1}, \ldots, F_{s}$ be a shelling order.

Assume there exists $d<a<s$, such that $F_{a}$ is not included in $\bigcup_{i<a} F_{i}$ but $F_{a+1}$ is. Then, $F_{1}, \ldots, F_{a-1}, F_{a+1}, F_{a}, F_{a+2}, \ldots, F_{s}$ is also a shelling order.

Proof. As $\left(\bigcup_{i<a} \overline{F_{i}}\right) \cap \overline{F_{a}}$ is a pure $d-2$ dimensional simplicial complex and as there exists $\alpha \in F_{a} \backslash \bigcup_{i<a} F_{i}$, we get that there exists $i<a$, such that $F_{a}-\alpha \subset F_{i}$. As $\left(\bigcup_{i<a} F_{i}\right) \cup F_{a+1}$ does not include $\alpha$, we get that $\left(\bigcup_{i<a} \overline{F_{i}} \cup \overline{F_{a+1}}\right) \cap \overline{F_{a}}$ is a pure $d-2$ simplicial complex.

Moreover, let $X$ be a face of both $F_{a+1}$ and $F_{j}(j<a)$, with dimension strictly less than $d-2$. Then, as $F_{1}, \ldots, F_{s}$ is a shelling order, there exists a $(d-2)$-dimensional face $Y \supseteq X$ which is a face of $F_{a+1}$ and $F_{i}$ (with $i<a+1$ ). If $i=a$ then, as $Y \subset F_{a+1}$, the vertex $\alpha$ does not belong to $Y$ and $Y=F_{a}-\alpha$. Hence, there exists $j<a$ such that $Y \subset F_{j}$. Altogether, $\left(\bigcup_{i<a} \overline{F_{i}}\right) \cap \overline{F_{a+1}}$ is a pure $(d-2)$-dimensional complex. 
Lemma 7. Let $\Delta$ be a pure $(d-1)$-dimensional abstract simplicial complex. If $\Delta$ is shellable, then it is vertex shellable.

Proof. Assume $\Delta$ is shellable. According to Lemma 6, there exists a shelling order $F_{1}, \ldots, F_{s}$ such that, for all $d<a<s$, if $F_{a}$ is not included in $\bigcup_{i<a} F_{i}$, no $F_{b}($ with $b>a)$ is included in $\bigcup_{i<a} F_{i}$. Hence, as $F_{i} \backslash\left(\bigcup_{j<i} F_{j}\right)$ includes at most one element, we can list the vertices of $\Delta$ the following way:

- begin with a list including the vertices of $F_{1}$ and let $a=1$.

- while $a<s$, add to the list the vertex (if any) of $F_{a+1}$ which is not in the list and let $a \leftarrow a+1$.

Eventually, we get a list $v_{1}, \ldots, v_{n}$ of all the vertices of $\Delta$, such that if $i<j$, all the $(d-1)$-faces of $\Delta$ with maximum element $v_{i}$ precedes all the $(d-1)$-faces including $v_{j}$ in the shelling order. Thus, we get:

$-\left\{v_{1}, \ldots, v_{d}\right\} \in \Delta$.

- for all face $\sigma \in \Delta$ with maximum vertex $v_{k}(k>d)$, there exists a face $\sigma^{\prime}$ that precedes $\sigma$ in the shelling order, such that $\sigma-v_{k} \in \sigma^{\prime}$. As $\sigma^{\prime}$ precedes $\sigma$ and does not include $v_{k}$, its maximal element is a vertex $v_{i}$ with $i<k$. Hence, the vertex $v_{j}$ such that $\sigma^{\prime}=\sigma-v_{k}+v_{j}$ is such that $j<k$.

- for all vertex $v_{k}(k \geq d)$, let $\Delta_{k}$ be the abstract simplicial complex $\Delta_{k}=$ $\left\{\sigma-v_{k}, \quad \sigma \in \Delta\right.$ and $\left.\sigma \subseteq\left\{v_{1}, \ldots, v_{k}\right\}\right\}$. Let $F_{a}$ and $F_{b}$ be the first and last $(d-1)$-dimensional face of the shelling order having $v_{k}$ as maximal element. We shall prove that $\Delta_{k}$ is a pure $(d-2)$-dimensional abstract simplicial complex having $F_{a}-v_{k}, \ldots, F_{b}-v_{k}$ as a shelling order: Consider in $\Delta_{k}$ a face $X$ of $F_{i}-v_{k}$ and $F_{j}-v_{k}$ with $a \leq i<j \leq b$. Then, $X+v_{k}$ is a face of $F_{i}$ and $F_{j}$ in $\Delta$. Thus there exists a $(d-2)$-dimensional face $Y \supseteq X+v_{k}$ and $h<j$, such that $Y \subseteq F_{h}$. As $v_{k}$ belongs to $F_{h}$, we get $h \geq a$. Hence, there exists a face $F_{h}-v_{k}$ of $\Delta_{k}$ having a $(d-3)$-dimensional face $Y-v_{k}$ including $X$ and included in $F_{j}$, what ends our proof.

Theorem 5. Let $\Delta$ be a pure $(d-1)$-dimensional abstract simplicial complex. Then, $\Delta$ is shellable if and only if it is vertex shellable.

Proof. According to Lemma 7, we only have to prove that the vertex shellability of $\Delta$ will imply its shellability.

Let $v_{1}, \ldots, v_{n}$ be a vertex shelling order. We list the $(d-1)$-faces of $\Delta$ the following way:

- let $F_{1}=\left\{v_{1}, \ldots, v_{d}\right\}$ and let $a=d$.

- while $a<s$ we add to the list the faces of $\Delta$ having $v_{k}$ as a maximal element in the order induced by the shelling order of $\Delta_{k}$.

Let $1 \leq i<j \leq s$ and let $X$ be a subset of $F_{i}$ and $F_{j}$ and let $v_{k}$ be the maximal element of $F_{j}$. 
- If $v_{k} \notin X$, then there exists a $(d-1)$-face $F_{h}$ including $F_{j}-v_{k}$ with $h<j$, according to the definition of a vertex shelling order. Obviously, $X \subseteq F_{j}-v_{k}$.

- If $v_{k} \in X$, then there exists a $(d-3)$-face $Y \supseteq X-v_{k}$ of $\Delta_{k}$ and a face $F_{h}$ having $v_{k}$ as a maximal element, such that $h<j$ and $F_{h}-v_{k} \supseteq Y$, according to the shelling order of $\Delta_{k}$. Hence, $Y+v_{k}$ is a $(d-2)$-face included in both $F_{h}$ and $F_{j}($ with $h<j$ ).

Let $R=\left(<_{1}, \ldots,<_{d}\right)$ be a $d$-representation. Let $X$ and $Y$ be two $(d-1)$ dimensional simplices of $\Sigma(R)$ and let $x_{i}$ (resp. $y_{i}$ ) be the maximal element of $X$ (resp. $Y$ ) with respect to $<_{i}$ (notice that $X=\left\{x_{1}, \ldots, x_{d}\right\}$ and $Y=$ $\left.\left\{y_{1}, \ldots, y_{d}\right\}\right)$, the $d$-order is the total order on the $(d-1)$-dimensional simplexes of $\Sigma(R)$ which is defined by:

$$
\forall X \neq Y \in \Sigma(R), \quad\left(X<Y \Longleftrightarrow x_{k}<_{k} y_{k}, \text { where } k=\min \left\{i, x_{i} \neq y_{i}\right\}\right)
$$

Theorem 6. Any standard abstract simplicial complex will be shellable.

More precisely, let $R=\left(<_{1}, \ldots,<_{d}\right)$ be a standard representation. Then, the $d$-order is a shelling order of $\Sigma(R)$ and any of the $<_{i}$ is a vertex-shelling order.

Proof. We shall only prove that the $d$-order is a shelling order, as this obviously implies that $<_{1}$ is a vertex shelling order (according to the definitions of a vertex shelling order and the fact that the $d$-order is a lexicographic order starting with $<_{1}$ ) and hence that $<_{i}$ is a vertex shelling order (by symmetry).

We shall prove that the $d$-order is a shelling order by induction over the dimension $d$ of the simplicial complex.

Let $F_{i}$ be the $i$ th face $(i>1)$ and let $x$ be its maximal element with respect to $<_{1}$. As $F_{1}$ is the only face including the $d-1$ exterior elements $v_{2}, \ldots, v_{k}$, there exists, according to Lemma 5 a maximal face $F_{j}$ including $F_{i}-x$ and which maximal element (with respect to $<_{1}$ ) is strictly smaller than $x$ and hence $j<i$. Let $F_{a}(a \leq i)$ be the first simplicial complex containing $x$, $\left(\bigcup_{j=1}^{a-1} \overline{F_{j}}\right) \cap \overline{F_{i}}=\overline{F_{i}-x}$ and, according to the induction on the dimension, if $a<i,\left(\bigcup_{j=a}^{i-1} \overline{F_{j}-x}\right) \cap \overline{F_{i}-x}$ is a pure simplicial complex of dimension $d-3$ and hence, $\left(\bigcup_{j=a}^{i-1} \overline{F_{j}}\right) \cap \overline{F_{i}}$ is a pure simplicial complex of dimension $d-2$. Altogether, $\left(\bigcup_{j=1}^{i-1} \overline{F_{j}}\right) \cap \overline{F_{i}}$ is a pure simplicial complex of dimension $d-2$.

Theorem 7. Let $\Delta$ be an abstract simplicial complex and $d=\operatorname{dim} P(\Delta)$. Then, there exists a standard d-representation $R=\left(<_{1}, \ldots,<_{d}\right) V(\Delta)$ defining a triangulation $\Delta^{+}$of $\Delta$, which is shellable and realizable in $\mathbb{R}^{d-1}$.

Proof. According to Theorem 4, $\Delta$ may be triangulated into $\Delta^{+}=\Sigma(R)$, where $R$ is a standard $d$-representation $V(\Delta)$. $\Delta^{+}$is shellable, according to Theorem 6 and its geometric realization follows from Theorem 3 , using any sufficiently fast increasing functions. 


\section{Acknowledgments}

The author would like to thank Jiri Matousek for his helpfull comments and remarks.

\section{References}

1. L. Barany, R. Howe, and H. Scarf, The complex of maximal lattice free simplices, Mathematical Programming 66 (Ser. A) (1994), 273-281.

2. D. Bayer, I. Peeva, and B. Sturmfels, Monomial resolutions, AMS electronic preprint \#199610-14-012, 1996, (a.k.a. alg-geom/9610012).

3. G. Brightwell and W.T. Trotter, The order dimension of convex polytopes, SIAM Journal of Discrete Mathematics 6 (1993), 230-245.

4. ㄴ The order dimension of planar maps, SIAM jouranl on Discrete Mathematics 10 (1997), no. 4, 515-528.

5. H. de Fraysseix and P. Ossona de Mendez, On topological aspects of orientations, Proc. of the Fifth Czech-Slovak Symposium on Combinatorics, Graph Theory, Algorithms and Applications, Discrete Math., (to appear).

6. - Regular orientations, arboricity and augmentation, DIMACS International Workshop, Graph Drawing 94, Lecture notes in Computer Science, vol. 894, 1995, pp. 111-118.

7. H. de Fraysseix, P. Ossona de Mendez, and P. Rosenstiehl, On triangle contact graphs, Combinatorics, Probability and Computing 3 (1994), 233-246.

8. H. de Fraysseix, J. Pach, and R. Pollack, Small sets supporting Fary embeddings of planar graphs, Twentieth Annual ACM Symposium on Theory of Computing, 1988, pp. 426-433.

9. How to draw a planar graph on a grid, Combinatorica 10 (1990), 41-51.

10. B. Dushnik, Concerning a certain set of arrangements, Proceedings of the AMS, vol. 1, 1950, pp. 788-796.

11. B. Dushnik and E.W. Miller, Partially ordered sets, Amer. J. Math. 63 (1941), 600-610.

12. T. Hibi, Algebraic combinatorics on convex polytopes, Carlslaw Publications, 1992.

13. G. Kant, Algorithms for drawing planar graphs, Ph.D. thesis, Utrecht University, Utrecht, 1993.

14. H. Scarf, The computation of economic equilibria, Cowles foundation monograph, vol. 24, Yale University Press, 1973.

15. W. Schnyder, Planar graphs and poset dimension, Order 5 (1989), 323-343. 